\title{
Modeling Contextual Concerns in Enterprise Architecture
}

\author{
Gonçalo Antunes*, José Barateiro*†, Christoph Becker*‡, José Borbinha* and Ricardo Vieira* \\ \{goncalo.antunes,jose.barateiro\}@ist.utl.pt, becker@ifs.tuwien.ac.at, \{jlb,rjcv\}@ist.utl.pt \\ *INESC-ID - Information Systems Group, Lisbon, Portugal \\ ${ }^{\dagger}$ LNEC, Lisbon, Portugal \\ $\ddagger$ Vienna University of Technology, Vienna, Austria
}

\begin{abstract}
Enterprise Architecture approaches are used to provide rigorous descriptions of the organization-wide environment, manage the alignment of deployed services to the organization's mission, end ensure a clear separation of the concerns addressed in an architecture. Thus, an effective Enterprise Architecture approach assists in the management of relations and dependencies of any components of the organization environment and supports the integration and evolution of the architecture. However, the quality of that approach is strongly influenced by the precision of the architecture context description, a fact which is not always recognized.

This paper focuses on the architecture context description and addresses the gap between the stakeholders' concerns and the resulting architecture. Based on a combination of established references and standards, we show how an explicit integration of the architecture context into the architecture model improves the linking of concerns and key elements of the architecture vision.

We apply our approach to a subject of increasing concern in the Information Systems area: longevity of information. Digital preservation is an interdisciplinary problem, but existent initiatives address it in a very domain-centric way, making it impossible to integrate documented knowledge into an overall organization architecture. We analyze several references and models and derive a description of the architecture context and a capability model that supports incremental development through an explicit distinction between systems and their capabilities.

The presented approach allows not just any organization to assess their current digital preservation awareness and evolve their architectures to address this challenge, but in particular demonstrates the added value of an explicit architecture context model in an Enterprise Architecture approach.
\end{abstract}

Index Terms-Enterprise Architecture; Capability; Digital Preservation; Architecture Context; Stakeholder Concerns;

\section{INTRODUCTION}

Architectures are an important tool for tackling the dynamic complexity of systems with diverse concerns and fostering communication between stakeholders [1]. They can be used to reach mutual understanding and consensus regarding the structure and behavior of a system and the rules governing it, both at the organizational and technological level. On the other hand, architectures provide a sound basis for documenting the concerns of the stakeholders, including architecture descriptions for multiple views of both functional and nonfunctional aspects of systems [2]. Enterprise Architecture (EA) approaches have received considerable attention due to their ability to align business and IT in organizations [3], which enables improved communication between executive stakeholders and IT personnel and makes way for the business vision to be reflected in operations and supporting systems [4].

However, due to the generic high-level nature of EA approaches, their tailoring and adaptation to specific domains is difficult and requires skilled and qualified resources both on EA and on the application domain. EA approaches generally prescribe models that do not necessarily express the organizational context or goals, requiring "situation-specific or adaptable solutions", which may limit the communication and interoperability with other components of the architecture [5].

The Open Group Architecture Framework (TOGAF) [6] is a high-profile EA framework. Its specification states the possibility to tailor both the process of creating and managing the architecture, as well as the architectural descriptions themselves, but no specific process or method for tailoring is provided. It only provides general high-level guidelines on terminology tailoring, process tailoring, and architecture content tailoring. In general, an EA provides a knowledge base to support the description of the architecture context, the tangible architecture definition, and the governance of the architecture. Generally, the real architecture definition is seen as the major artifact and tangible result of an EA. However, its quality is strongly influenced by the precision of the architecture context description, a fact which is not explicitly recognized in the models of leading frameworks.

This paper shows how a combination of TOGAF with other established references and standards can improve the architecture context description by explicitly addressing the gap between stakeholders' concerns and the resulting architecture. A fusion of approaches enables interdisciplinary business-IT alignment in the Architecture Vision of the TOGAF architecture development method and supports the communication between involved stakeholders, from business owners to solution providers, through the explicit consideration of domain concerns within the EA process.

We thus discuss the product of applying our EA approach to the digital preservation domain. Digital preservation (DP) can be seen as a problem of IT/Business alignment, embodied in specific enterprise contexts. In order to preserve digital objects, technology should provide adequate support to assure the integrity, authenticity, and understandability of objects through time, in a changing technological landscape. 
It is recognized that DP solutions must always be a mix of organizational structure with the related set of activities and services, supported by an adequate IT infrastructure aligned with the DP vision. As a consequence of our work, we present a detailed description of a general architecture context with DP capabilities, which can be extended to accommodate scenario specific requirements and/or constraints.

The remainder of this paper is organized as follows. Section II describes important standards, models and references that form the basis of our work. Section III describes our approach to developing the description of the architecture context that explicitly incorporates domain concerns into the architecture. Section IV presents the results of applying TOGAF's Preliminary Phase to scope the DP architecture context, while Section V describes the respective architecture vision with DP capabilities. Finally, Section VI draws conclusions and gives an outlook on current and future work.

\section{RELATED WORK}

This section describes important concepts forming the foundation for this work. We describe the IEEE Std. 1471-2000, TOGAF, the Business Motivation Model, and the DP domain and previous approaches to analyze it.

\section{A. IEEE Std. 1471-2000}

Architectural descriptions provide rigorous descriptions of complex systems with diverse concerns, and are a recommended approach to tackling the dynamic and increasing complexity of those systems. An architecture has to reflect the concerns and interests of the stakeholders. It can also be described as "a vehicle for communication and negotiation among stakeholders" [1]. Taking that into account, an architecture must also reflect the different viewpoints of all the interested parts, so that it can be communicated efficiently. A stakeholder is a viewer that perceives and conceives the universe, using his/her senses, in order to produce conceptions resulting from the interpretation of what is observed [1]. A viewer can form a representation of the conceptions he/she makes using a determined language to express himself. When observing the universe, a viewer will be interested only in a specific subset of that universe, which is called a concern. The conceptualization of that subset of the universe is called a domain.

According to the IEEE Std. 1471-2000, which has also become ISO/IEC 42010:2007, architecture is "the fundamental organization of a system, embodied in its components, their relationships to each other and the environment, and the principles governing its design and evolution" [7]. It considers that a system has a mission and inhabits an environment which influences it. It also has one or more stakeholders that have concerns regarding the system and its mission. Concerns are "those interests that pertain to the system's development, its operation, or any other aspects that are critical or otherwise important to one or more stakeholders". A system has an architecture described by an architecture description which includes a rationale for the architecture. The architecture description is also related with the stakeholders of the system and deals with several views according to the viewpoints of the stakeholder. This includes functional and non-functional aspects of stakeholders' concerns. Accurate architecture descriptions provide a "complete picture" of the overall system. However, any system (especially a complex system made of software, people, technology, data and processes) is continuously subject to changes, usually driven by the evolution of the system environment [2].

\section{B. TOGAF}

EA is a holistic approach to systems architecture with the purposes of modeling the role of information systems and technology on the organization, aligning enterprise-wide concepts, business processes and information with the information systems, planning for change, and providing self-awareness to the organization [6].

The Open Group Architecture Framework (TOGAF) [6] is a high-profile EA, providing methods and tools to support architecture development. It comprises seven modules which can be partly used independently of each other. The core of TOGAF are the Architecture Development Method (ADM) and the Architecture Content Framework.

The ADM as the heart of TOGAF consists of a cyclical process divided in nine phases. After a preliminary phase in which the context, relevant guidelines and standards and the goals of the architecture process are identified, the main process begins with the elaboration of an architecture vision and the principles that should guide the architecture. This provides the basis for subsequent phases: business architecture, information systems architecture, and technology architecture. On this basis, solutions are developed (opportunities and solutions phase), and migration and implementation are planned and governed (migration planning and implementation governance phases). Finally, architecture change management ensures that the architecture continues to be fit for purpose. The ADM can be adapted for various purposes, and in more complex situations, the architecture can be scoped and partitioned so that several architectures can be developed and later integrated using an instance of the ADM to develop each one of them.

The Architecture Content Framework is TOGAF's own architecture framework. It specifies the different types of architecture products and provides a Content Metamodel which can be related with the phases of the ADM. The Content Metamodel features a detailed definition of the business entities. No relations are made within the entities associated with the Architecture Vision and Preliminary phases. The elements defined in the Meta-model should be used in the definition of viewpoint models, may it be the ones suggested by TOGAF or customized viewpoints.

\section{Business Motivation Model}

The Content Metamodel included in TOGAF provides entities associated with the description of the architectural context leading to the development of a vision for the architecture. However, the Content Metamodel lacks high-level concepts to 
allow explicit tracing from the concerns of the stakeholders to the means an organization should employ to meet its ends.

The Business Motivation Model (BMM) [8] provides a conceptual framework for the development, communication, and management of business plans, identifying motivational factors behind it, the elements required for its formulation, and relationships between the elements.

Four major concepts are defined: End, Mean, Influencer, and Assessment. An End is "what an enterprise wants to be". Ends can be the Vision for the organization (what the organization wants to be); or a Desired Result, which can be either a Goal (long-term, comprised of Objectives) or an Objective (short-term, component of Goals). A Mean is "what an enterprise has decided to do in order to become what it wants to be", in other words, to achieve its Ends. Means can be the Mission, which describes what an organization does in order to achieve the Vision; Courses of Action, which can be a Strategy or Tactic; and Directives, which govern the Courses of Action. An Influencer is "something that can cause changes that affect the enterprise in its employment of its Means or achievement of its Ends". An Influencer can be External (from outside the organization; e.g., Applicable Legislation) or Internal (from within the organization; e.g., Available Resources). An Assessment is "a judgment about the influence" of an Influencer "on the enterprise's ability to employ its means or achieve its ends", with decisions stemming from that being reflected in changes to the Ends and/or Means.

DP can be seen as a problem of IT/Business alignment where deployed means (e.g., the support technology where information resides) are often set to meet ends other than the concern of keeping information accessible and understandable. The next section will shortly outline the key problem areas and concepts of this relatively new field.

\section{Digital Preservation}

Physical artifacts like printed works or paintings can survive for centuries in controlled conditions. In contrast, digital objects inhabit an unstable environment and require the execution of continuous actions to make it possible to interpret them in the future. DP aims at optimizing the information life-cycle management from the creation to the dissemination and use of information objects to maintain the knowledge contained in these objects accessible beyond the limits of media failure or technological change, ensuring authenticity and integrity [9].

DP is an issue caused by the fact that IT solutions are inherently short-lived and ever-changing, causing organizational knowledge to be trapped and confined in obsolete or proprietary formats, in a context where IT problems and solutions intersect with organizational policies and missions. The complexity of DP increases with the fact that each organizational scenario contains different types of digital objects, each having its own specific requirements. For instance, the preservation of digital objects with static content (e.g., text files) is relatively straightforward, as opposed to the preservation of objects with dynamic behavior (e.g., a software synthesizer). Furthermore, semantics might be context-dependent and not fully included in the objects, so that they require a determined environment (e.g., web content in a web browser). The problem increases with the preservation of multiple inter-dependent objects, which with the loss of any constituent object might be impossible to interpret.

Although DP presents a problem faced by all type of organizations that have to manage information and use technology to support their business processes, initiatives have been pushed largely by cultural heritage institutions [10]. In general, efforts focus on the design and implementation of trustworthy digital repositories for archiving information. The Open Archival Information System (OAIS) Reference Model [11] is a model of concepts and functional entities with the aim to provide a common language for the domain and guide the design of preservation systems. The OAIS provides a highlevel and narrow view of the main functions of a preservation system and prescribes a certain solution architecture that might not be adequate for determined organizations. Several other important references of the domain exist, such as criteria catalogs specifying requirements that a repository should fulfill [12][13]. Though all these references are of tremendous value for the preservation community, they suffer from internal inconsistencies and lack conceptual alignment between them.

A previous approach on modeling context in DP is presented in [14], although it focuses on the life cycle context of preservation systems, with the aim of representing the production and reuse context of digital content, leaving out the remaining business context. Some contributions have addressed DP from an Information Systems perspective. In [15], the authors present an approach which applies IBM Component Business Modeling to a DP scenario to identify services needed to perform DP in a digital repository. Additionally, the technology existing on the organization can be assessed for its capability to deliver those services. The authors in [16] analyzed the domain using Risk Management terminology, defining a taxonomy of threats and vulnerabilities with a holistic view on DP. A generic TOGAF-based Reference Architecture was presented in [17] which presented an important step towards a more holistic view on the DP problem, based on EA concepts. However, it was not based on an in depth analysis of existing domain knowledge bases to a degree that enables their convergence in a transparent manner. In that sense, a consistent approach to the problem is still to be found.

\section{Developing the Architecture Context}

The adoption of an EA approach, and the corresponding separation of concerns, results in a common understanding of the architecture with controlled complexity, reducing the risks of the architecture design [18]. Moreover, the very objective of EA, i.e. strategic alignment, is critical to DP success. The lack of alignment existing in the various DP reference models is one of the key inhibitors of advances in the State of Art on the subject, as standardization efforts are vague and hardly applicable to specific scenarios, and decision making models and traceability are scarce. 
By projecting the domain-specific knowledge bases against the backdrop of an established EA framework, we can establish the boundaries between the domain and generically applicable concerns, scope the problem definition of the domain and improve strategic alignment and traceability through the explicit clarification of capabilities. Such an architecture will provide organizations with a tool to add DP capability to any business governing data that is motivated to address the concern of DP.

We are using TOGAF's ADM to develop a vision for the architecture which accommodates domain-specific capabilities. The purpose is to develop a capability-centered reference architecture that addresses domain-specific concerns and integrates reference models from a specific domain. We thus discuss the accommodation of domain-specific concerns in the two key phases that ADM commences with: Preliminary and Architecture Vision.

The Preliminary phase of the ADM consists of the preparation and initiation of the architectural activities and includes the definition of the principles that will govern the architecture work. During this phase, the internal and external organizations impacted by the architecture work are assessed and defined, and the key reference models established. Subsequently, key references are analyzed and merged in order to create a general understanding of the domain. By reconciling and harmonizing concepts and terminology of different sources, the proposed approach enables the reuse of best practices, improves common understanding and preserves this knowledge for future projects. That understanding creates the conditions to initiate the Architecture Vision phase in which key concepts for a DP architecture emerge.

The Architecture Vision phase includes the definition of scope, the identification of stakeholders and their concerns and the elaboration of a value chain; constraints, drivers, goals, and key performance indicators; and finally, capabilities and the envisioned solution architecture. The content metamodel of TOGAF defines entities associated with the Architecture Vision development, but no explicit relationships are made between these entities. Some entities that are central to the Architecture Vision and used in several artifacts resulting from this phase are not explicitly modeled in the metamodel (e.g., Stakeholder and Concern, which are as well main concepts of the IEEE Std. 1471-2000 [7]). The Motivation extension of the metamodel provides additional entities such as Driver, Goal, and Objective in order to "allow additional structured modeling of the drivers, goals, and objectives that influence an organization to provide business services to its customers" [6]. However, those entities are depicted as belonging to the Business Architecture, thus being explicitly modeled during its development. Additionally, no explicit relationships are detailed between those entities and the ones allegedly pertaining to the Architecture Vision.

Explicitly taking these contextual concerns into account as part of the Architecture Vision enables us to better address IT/Business alignment by improving the traceability between strategic vision and mission and the concrete systems im-

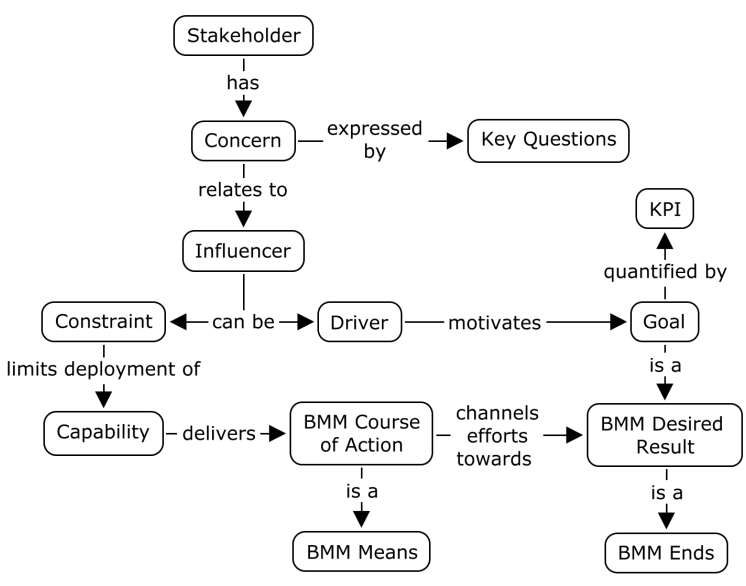

Fig. 1. Main concepts of the architecture vision

plementation. In order to achieve that architecture vision, we merged concepts from the IEEE Std. 1471-2000 and the Business Motivation Model into TOGAF. Figure 1 depicts the main concepts used in developing a architecture vision, while Table I provides the description of the concepts.

\section{Scoping the Digital Preservation Domain}

Several reference models of the DP domain and from other related domains were analyzed including: the Open Archival Information System (OAIS) Reference Model [11]; ISO 20652 - Space data and information transfer systems - Producerarchive interface - Methodology abstract standard [19]; Trusted Digital Repositories: Attributes and Responsibilities [20]; PREservation Metadata: Implementation Strategies (PREMIS) Data Dictionary [21]; Trustworthy Repository Audit and Certification Criteria and Checklist (TRAC) [12]; Preservation Planning [22]; SHAMAN Reference Architecture [17]; Model requirements for Electronic Records Management (MoReq) 2010 [23]; Control OBjectives for Information and related Technology (COBIT) [24]; IEEE Std. 1540-2001 - Standard for Software Life Cycle Processes - Risk Management [25]; and ISO/IEC 27001:2005 Information technology - Security techniques - Information security management systems - Requirements [26].

These reference models typically contain: (i) Description of typical stakeholder concerns, actors and their goals and interests; (ii) Mandatory requirements and constraints necessitated by commonly encountered external drivers, legal obligations or other non-negotiable requirements; (iii) Contracts and governance metrics; (iv) Domain concepts and corresponding design patterns for domain models, roles and interactions; $(v)$ Design patterns and building blocks for solutions; and (vi) Value propositions for functions and systems with or without reference to actors or stakeholders. These aspects need to be considered at different stages of the architecture development cycle. In addition, domain references may contain statements that can be interpreted in different ways and span different architecture concerns. Merging disparate sources is only feasible based on a clear distinction between these categories of statements and on clear definitions of the used terminology. 
TABLE I

DESCRIPTION OF THE MAIN CONCEPTS OF THE ARCHITECTURE VISION

\begin{tabular}{|l|l|}
\hline Concept & Definition \\
\hline Stakeholder & An individual, team, or organization (or classes thereof) with interests in, or concerns relative to, a system [7] \\
\hline Concern & $\begin{array}{l}\text { Concerns are those interests which pertain to the system's development, its operation or any other aspects that are critical or } \\
\text { otherwise important to one or more stakeholders [7] }\end{array}$ \\
\hline Key Questions & Stakeholder-made questions to express their concerns [6] \\
\hline Influencer & Something that can cause changes that affect the enterprise in its employment of its Means or achievement of its Ends [8] \\
\hline Driver & An external or internal condition that motivates the organization to define its goals [6] \\
\hline Goal & A high-level statement of intent or direction for an organization. Typically used to measure success of an organization [6] \\
\hline $\begin{array}{l}\text { Key Performance } \\
\text { Indicator }\end{array}$ & A way of quantifying the performance of the business or project [6] \\
\hline Constraint & An external factor that prevents an organization from pursuing particular approaches to meet its goals [6] \\
\hline Capability & $\begin{array}{l}\text { An ability that an organization, person, or system possesses. Capabilities are typically expressed in general and high-level terms } \\
\text { and typically require a combination of organization, people, processes, and technology to achieve [6] }\end{array}$ \\
\hline $\begin{array}{l}\text { BMM Course of } \\
\text { Action }\end{array}$ & $\begin{array}{l}\text { is a way of configuring some aspect of the enterprise (things, processes, locations, people, time) to channel efforts towards Desired } \\
\text { Results [8] }\end{array}$ \\
\hline BMM Means & Means are about what an enterprise has decided to do in order to become what it wants to be [8] \\
\hline BMM Ends & Ends are about what an enterprise wants to be [8] \\
\hline $\begin{array}{l}\text { BMM Desired } \\
\text { Result }\end{array}$ & A Desired Result is an End that is a state or target that the enterprise intends to maintain or sustain [8] \\
\hline
\end{tabular}

We have relied on several techniques to facilitate contextualization and alignment of knowledge bases. One of these techniques used the Zachman framework [27], which intends to address a holistic view of the organization, to map the concepts of the domain references, in order to develop an understanding of the concerns covered. Concept maps were also an employed technique, since they are both a practical tool for isolating and visualizing concepts and relationships. Through the application of this technique to the domain reference models, it was possible to detect misalignments between the concepts used on references of the same domains. More details on the use of these techniques are provided in [28].

\section{AN ARCHITECTURE VISION WITH DP CAPABILITIES}

In this section we describe the identified DP stakeholders and their concerns, driver categories, high-level goals, performance indicators, and capabilities. We also describe the resulting architecture vision.

\section{A. Stakeholders and Concerns}

One of the first steps in the development of an architecture vision is the identification of stakeholders, their key questions, and the elaboration of their concerns. The identification of stakeholders should take into account users of the system, those responsible for the acquisition and governance of the system, the developers or providers of the components of the system, and those responsible for the maintenance of the system [7]. Twelve stakeholders were identified:

- Producer/Depositor - The entity responsible for the ingestion of the objects to be preserved. It may be the owner of the object, but can also be any other entity entitled to perform this action. The terms Producer and Depositor are used interchangeably in distinct sources to describe the stakeholder responsible for content ingestion.

- Consumer - Represents the user accessing the preserved objects, who has a potential interest in its reuse and a certain background in terms of knowledge and technical environment.
- Executive Management - Is responsible for strategic decision making on an organization level, ensuring that the mandate is fulfilled and the repository continues to serve its designated community.

- Repository Manager - Ensures repository continuity, defines strategies, sets goals and objectives to be achieved by the repository and thus operates on the business domain, interacting with the designated communities and the legal environment.

- Technology Manager - Is responsible for technological system continuity and the deployment of technological means to achieve the ends set by the repository business.

- Operational Manager - Is concerned with the continuous policy-compliant operation of the repository, which involves balancing ends and means and resolving conflicts between them, i.e. constraints as set from Technology Manager and Repository Manager.

- Regulator - An external entity imposing rules concerning the preservation of digital assets, such as legislation and standards. These can apply to the organization or the system's technology and usage.

- Auditor - Responsible for certifying that the organization practices, the system's properties and the operational environment comply with established standards and regulations.

- Repository Operator - A business worker who may be aware of the details of the design and deployment of the system, but is primarily concerned with business, with no concerns about infrastructure management or strategic alignment.

- Technology Operator - Is responsible for the regular operation and maintenance of the components of the technical infrastructure (hardware and software) and their interoperability, according to specified service levels.

- System Architect - Is responsible for the design and update of the architecture of the system and its alignment with the business objectives.

- Solution Provider - Is concerned with providing com- 
ponents of the architecture. This may include software components, platforms and business services.

The key questions posed by stakeholders are an indication of their core interests. Organizing these interests leads to the definition of concerns. Table II presents example key questions and concerns for the stakeholder Executive Management.

\section{B. Influencers}

Through the analysis of the concerns of the stakeholders, it becomes possible to analyse the main influencers that have an impact on the setting of organizational goals in DP. The result of this analysis is a categorization of DP drivers and an assessment of possible constraints (through external drivers).

Drivers can be Internal or External. The top-level Internal Driver categories are: Business Vision, which can be considered the fundamental driver of the organization; Resources, which consists of the organizational and technical infrastructure required to operate; Data, which is represented by the characteristics of the data to be preserved by the organization; and Processes, which is related to the characteristics of the processes manipulating data.

The top-level External Driver categories are: Producers of information, which influence the definition of the objectives of the organization through the technology they use to produce data, the data contents produced by them, the need to satisfy their demands, and the need to build trust and reputation among them; User Community, which influences the definition of the objectives of the organization through the technology they use to access and use the data, the knowledge needed to understand the informational content of the objects, the need to satisfy their demands, and the need to build trust and reputation near them; Contracts, such as Deposit, Supplier and Service, Interoperability, and Access Contracts; Supply of Technology, Services and People; Competition caused by overlaps with other organizations, which may require the organization to differentiate its services; and finally, Regulation and Mandate. The latter includes Regulatory/Legal Constraints, the Internal regulation of the organization, the Mandate to preserve contents, Rights and Ownership concerning the objects to be preserved, the possible existence of Certification and a corresponding motivation to be certified, and the need for sufficient funding.

This categorization of drivers can also be used to assess constraints. For instance, considering the external driver category Funding, a possible constraint posed by this type of driver could be Insufficient funding constrains the deployment of capability " $Y$ " on the organization.

\section{Goals and Key Performance Indicators}

The analysis completed to this point allows the definition of high-level goals for DP. The performance of an organization towards the achievement of defined goals can be assessed trough the measure of performance indicators.

Four goals are related with the primary vision of the organization: (i) G1 - Acquire content from producers in accordance to the mandate, following agreed rules. An example Key
Performance Indicator (KPI) for assessing this goal would be "Number of distinct objects received per year"; (ii) G2 Deliver authentic, complete, usable and understandable objects to designated user community. An example KPI for assessing this goal would be "Percentage of transformational object properties preserved by actions as denoted by user feedback and/or QA measures in comparison to guarantees provided by specified SLAs"; (iii) G3 - Faithfully preserve provenance of all objects and deliver accurate provenance information to the users upon request. An example KPI for assessing this goal would be "Percentage of access requests where objects' provenance is reported to be undefined, not clearly defined or wrong (e.g. indicated by number of incidents of fake objects reported)"; (iv) G4 - Authentically preserve objects for the specified time horizon, keeping their integrity and protecting them from threats. An example KPI for assessing this goal would be "Percentage of legitimate access requests fulfilled successfully as denoted by user feedback".

Three other goals relate to continuity in changing environments: (i) G5 - React to changes in the environment timely in order to keep objects accessible and understandable. An example KPI for assessing this goal would be "Average reaction time responding to obsolescence incident report"; (ii) G6 Ensure repository sustainability: mandate, technical, financial, operational, communities. An example KPI for assessing this goal would be "Time horizon of secured mandate greater or equal to average time horizon of objects"; (iii) G7 - Build trust in the depositors, the designated community and other stakeholders. An example KPI for assessing this goal would be "Time horizon of mandate secured by legal means in years".

Finally, one goal is related with efficiency: G8 - Maximize efficiency in all operations. An example KPI for assessing this goal would be "Average yearly costs per object".

\section{Capabilities}

Capabilities are expressed in terms of its outcome, in a general and high-level manner. A capability is not a business function, but an ability realized by a combination of elements such as actors, business functions and business processes, and technology. The capabilities of the scenario at hand can be divided into governance capabilities, business capabilities, and support capabilities.

Governance capabilities include: (i) Compliance, which is the ability to verify the compliance of operations and report deviations, and is related to goals G6 and G7; (ii) Community Relations, which is the ability to engage with the designated community and ensure that its needs are fulfilled, and is related to goals G5 and G7; (iii) Certification, which is the ability to obtain and maintain certification status, and is related to goals G6 and G7; (iv) Mandate Negotiation, which is the ability to negotiate mandates with governing institutions in scenarios where a legal mandate is present, and is related to goals G6 and G7; (v) Business Continuity, which is the ability to identify business capabilities and assure mission-critical operations, and is related to goals G5 and G6; (vi) Succession Planning, which is the ability to negotiate formal succession plans and 
TABLE II

CONCERNS AND KEY QUESTIONS FOR THE EXECUTIVE MANAGEMENT STAKEHOLDER

\begin{tabular}{|l|l|}
\hline Concern & Key Questions \\
\hline $\begin{array}{l}\text { Mandate, mission, policies and compliance: The governance } \\
\text { of the mandate, the commitment of the organization to DP, } \\
\text { may it be for business needs, legal, or legislative reasons; } \\
\text { and corresponding compliance. This includes certification and } \\
\text { succession planning }\end{array}$ & $\begin{array}{l}\text { Is the mandate adequate, well-specified and appropriately accessible? Is the organiza- } \\
\text { tion able to fulfill the mandate? Does the organization possess all required contracts } \\
\text { regarding succession planning and escrow agreements? Is the organization compliant } \\
\text { to external regulations? Does the organization possess necessary certifications? }\end{array}$ \\
\hline $\begin{array}{l}\text { Roles and Responsibilities: Assignment of roles and respon- } \\
\text { sibilities to staff needed to perform the required roles in order } \\
\text { to fulfill the preservation responsibilities }\end{array}$ & $\begin{array}{l}\text { Have we defined all responsibilities? Do we have roles addressing all responsibilities? } \\
\text { Do we have qualified staff assigned to fill each role? }\end{array}$ \\
\hline $\begin{array}{l}\text { Financial Sustainability: Ensuring the continued sustainability } \\
\text { of the preservation business }\end{array}$ & Is the organization financially sustainable for the time horizon of the mandate? \\
\hline $\begin{array}{l}\text { Strategic planning: Development of repository strategies and } \\
\text { definition of goals to ensure strategic alignment and business } \\
\text { continuity }\end{array}$ & $\begin{array}{l}\text { What are the goals and objectives that best represent our vision? Which strategies are } \\
\text { optimal to achieve our goals and objectives? }\end{array}$ \\
\hline
\end{tabular}

is related to goals G6 and G7; (vii) IT Governance, which is the ability to manage and develop the services, processes and technology solutions that realize and support the primary capabilities, and is related to goals G5, G6 and G8; and (viii) Manage Risks, which is the ability to manage and control strategic and operational risks and opportunities to ensure efficient business continuity and sustainability, and is related with goals G6 and G8.

Business capabilities include: (i) Acquire Content, which is the ability to offer services for transferring content from producers into the repository, including services for reaching agreement with producers about the terms and conditions of transfer. It is realized by the component capabilities Ingest and Ingest Negotiation, and is related to goal G1; (ii) Secure Bitstreams, which is the ability to secure bitstreams for a specified amount of time (Bitstream preservation). It is realized by Bitstream Security Planning and Secure Storage Operation, and is related with goals G3 and G4; (iii) Preserve Content, which is the ability to maintain content authentic and understandable to the defined user community over time and assure its provenance (Logical preservation). It is arguably the core DP capability and is realized by Preservation Planning and Preservation Operation. It is related with goals G3, G4, and G5; and (iv) Disseminate Content, which is the ability to offer services for delivering content contained in the repository to the user community or another repository, including services for reaching agreement with users about the terms and conditions of delivery. It is realized by Discovery, Access, and Dissemination Negotiation, and is related with goal G2.

Finally, Support capabilities include: (i) Data Management, which is the ability to manage and deliver data management services, i.e. to collect, verify, organize, store and retrieve data (including metadata) needed to support the preservation business according to relevant standards. It is related with goals G2 and G3; (ii) Manage Infrastructure, which is the ability to ensure continuous availability and operation of the physical, hardware, and software assets necessary to support the repository. It is related with goals G5, G6, and G8; (iii) Manage $H R$, which is the ability to continuously maintain staff which is sufficient, qualified and committed to performing the tasks required by the repository. It is related with goals G6 and G8; and (iv) Manage Finances, which is the ability to plan, control and steer financial plans and operations of the repository to ensure business continuity and sustainability. It is related with goals G6 and G8.

In a business environment, DP will generally be seen as a part of IT Governance. Since it is our core focus of modeling, DP is highlighted and as such presented separately from more general aspects of IT Governance. Upon realization in a business environment, DP (and Data Management) will likely be realized as part of IT Governance, and will thus be submerged into it.

Through the definition of capabilities, it becomes possible to trace back into the goals and drivers of the organization. Since each organization is different in many particular aspects, some capabilities might not apply, while others will be required.

The presented capabilities provide a wide coverage of the organization. Table III depicts the mapping of the described capabilities in the Zachman framework. The Compliance and Risk Management capabilities cover all cells of the Zachman framework, therefore are omitted to improve readability.

\section{CONCLUSION}

This paper discusses the problem of describing an architecture context within EA practice. The quality of the architecture context description is crucial to achieve optimal results, since subsequent stages of evolving EA descriptions heavily depend on the architecture context. The context thus has a strong impact on the achievement of strategic alignment between high level strategies and implemented services.

We showed how a combination and reconciliation of several standards and best-practices and a normalized metamodel to describe an architecture context enables separation of concerns and common understanding of potentially conflicting domainspecific knowledge sources or stakeholders' view of the systems. We present a combination of concepts from TOGAF with the IEEE Std. 1471-2000 and the Business Motivation Model. This leads to a contextualised architecture vision that explicitly incorporates the concerns of stakeholders.

We illustrated the added value of this architecture context in an application to DP. This emerging interdisciplinary domain poses challenges to several communities, but is currently still addressed in a domain-centric fashion not aligned with the enterprise environment. Addressing DP concerns in isolation, while ignoring EA, can easily conflict with other concerns 
TABLE III

CAPABILITY MAPPING ON THE ZACHMAN FRAMEWORK

\begin{tabular}{|c|c|c|c|c|c|c|}
\hline & data & function & network & people & time & motivation \\
\hline scope & $\begin{array}{l}\text { IN, DN, MN, } \\
\text { C, SP }\end{array}$ & $\mathrm{IN}, \mathrm{DN}, \mathrm{BC}$ & $\mathrm{IN}, \mathrm{DN}, \mathrm{SP}$ & $\begin{array}{l}\mathrm{IN}, \mathrm{DN}, \mathrm{CR}, \\
\mathrm{MN}, \mathrm{C}, \mathrm{SP}\end{array}$ & $\begin{array}{l}\text { IN, DN, MN, } \\
\text { C, SP, BC }\end{array}$ & $\begin{array}{l}\text { IN, DN, CR, } \\
\mathrm{MN}, \mathrm{C}, \mathrm{BC}\end{array}$ \\
\hline business & $\mathrm{I}, \mathrm{D}, \mathrm{A}, \mathrm{PP}, \mathrm{DM}$ & $\begin{array}{l}\text { I, D, A, PP, } \\
\text { BSP, DM, ITG }\end{array}$ & I, A, ITG & ITG & ITG & PP \\
\hline system & $\mathrm{I}, \mathrm{D}, \mathrm{A}, \mathrm{PP}, \mathrm{DM}$ & $\begin{array}{l}\mathrm{I}, \mathrm{D}, \mathrm{A}, \mathrm{PP}, \\
\text { BSP, DM, ITG, } \\
\text { PO, SSO }\end{array}$ & I, A, ITG, SSO & ITG & ITG & PP \\
\hline technology & $\begin{array}{l}\text { PP, PO, SSO, } \\
\text { BSP, DM }\end{array}$ & $\begin{array}{l}\text { PP, PO, SSO, } \\
\text { BSP, DM, ITG }\end{array}$ & ITG, SSO, BSP & ITG & ITG & PP \\
\hline components & $\mathrm{PO}, \mathrm{SSO}, \mathrm{DM}$ & $\mathrm{PO}, \mathrm{SSO}, \mathrm{DM}$ & SSO, ITG & ITG & ITG & $\mathrm{PO}$ \\
\hline
\end{tabular}

Legend: I: Ingest; IN: Ingest Negotiation; BSP: Bitstream Security Planning; SSO: Secure Storage Operation; PP: Preservation Planning; CR: Community Relations; MN: Mandate Negotiation; BC: Business Continuity; SP: Succession Planning; and ITG: IT Governance. PO: Preservation Operation; D: Discovery; A: Access; DN: Dissemination Negotiation; DM: Data Management; C: Certification;

of an organization. We showed how DP concerns can be integrated in EA efforts. We presented key artifacts that result from a harmonization of DP models and EA best practices. We discussed the main artifacts of the architecture context applied to DP, including Stakeholders and their Concerns; Influencers (drivers and constraints); Goals and KPIs; a Capability model; and a Solution Concept providing a homogeneous vision of the overall architecture to different stakeholders.

After the assessment, the development and deployment of capabilities in concrete scenarios becomes possible through the development of architecture viewpoints, following the TOGAF ADM Business Architecture phase. The viewpoints should address the concerns of stakeholders with a special interest in a determined capability. Viewpoints should provide a means to model the change needed, providing the architecture artefacts needed to guide the development of the capabilities in the organization.

\section{ACKNOWLEDGMENT}

This work was supported by FCT (INESC-ID multiannual funding) through the PIDDAC Program funds and by the project SHAMAN (Sustaining Heritage Access through Multivalent Archiving), partially funded by the EU under the FP7 contract $n^{\circ} 216736$.

\section{REFERENCES}

[1] H. A. Proper, A. A. Verrijn-Stuart, and S. J. B. A. Hoppenbrouwers, "On utility-based selection of architecture-modelling concepts," in Proceedings of the 2nd Asia-Pacific conference on Conceptual Modelling, 2005.

[2] T. Mens, J. Magee, and B. Rumpe, "Evolving software architecture descriptions of critical systems," Computer, vol. 43, pp. 42-48, 2010.

[3] I. Motjolopane and I. Brown, "Strategic business-it alignment, and factors of influence: a case study in a public tertiary education institution," in Proc. SAICSIT '04, 2004. [Online]. Available: http://portal.acm.org/citation.cfm?id=1035053.1035071

[4] W. V. Grembergen, Strategies for information technology governance. Idea Group Publishing, 2004.

[5] J. Saat, U. Franke, R. Lagerstrom, and M. Ekstedt, "Enterprise architecture meta models for it/business alignment situations," in 14th IEEE International Enterprise Distributed Object Computing Conference, 2010.

[6] The Open Group, TOGAF Version 9. Van Haren Publishing, 2009.

[7] IEEE, Recommended Practice for Architecture Description of SoftwareIntensive Systems (IEEE 1471-2000), IEEE Computer Society, 2000.

[8] Object Management Group, Business Motivation Model 1.1. OMG, May 2010.
[9] M. Jones and N. Beagrie, Preservation Management of Digital Materials: A Handbook. London, UK: Digital Preservation Coalition, November 2008. [Online]. Available: http://www.dpconline.org/graphics/handbook/index.html

[10] C. Webb, Guidelines for the Preservation of Digital Heritage. Information Society Division United Nations Educational, Scientific and Cultural Organization (UNESCO) - National Library of Australia, 2005.

[11] ISO, Open archival information system - Reference model (ISO 14721:2003), 2003.

[12] CRL and OCLC, "Trustworthy Repositories Audit \& Certification: Criteria and Checklist (TRAC)," The Center for Research Libraries and Online Computer Library Center, Tech. Rep., February 2007. [Online]. Available: www.crl.edu/PDF/trac.pdf

[13] S. Dobratz, A. Schoger, and S. Strathmann, "The nestor catalogue of criteria for trusted digital repository evaluation and certification," in Proc. JCDL2006, 2006.

[14] H. Brocks, A. Kranstedt, G. Jäschke, and M. Hemmje, Smart Information and Knowledge Management, ser. Studies in Computational Intelligence. Springer Berlin / Heidelberg, 2010, ch. Modeling Context for Digital Preservation, pp. 197-226.

[15] R. J. van Diessen, B. Sierman, and C. A. Lee, "Component business model for digital repositories: A framework for analysis," in Proc. iPRES 2008, 2008.

[16] J. Barateiro, G. Antunes, F. Freitas, and J. Borbinha, "Designing digital preservation solutions: A risk management-based approach," International Journal of Digital Curation, vol. 5, no. 1, 2010.

[17] G. Antunes, J. Barateiro, and J. Borbinha, "A reference architecture for digital preservation," in Proc. iPRES2010, Vienna, Austria, 2010.

[18] R. Cloutier, G. Muller, D. Verma, R. Nilchiani, E. Hole, and M. Bone, "The concept of reference architectures," Systems Engineering, vol. 1, no. 13, pp. 14-27, 2010.

[19] ISO, Space data and information transfer systems - Producer-archive interface - Methodology abstract standard (ISO 20652:2006), 2006.

[20] RLG/OCLC Working Group on Digital Archive Attributes, Trusted Digital Repositories: Attributes and Responsibilities. Research Libraries Group, 2002.

[21] PREMIS Editorial Committee, PREMIS Data Dictionary for Preservation Metadata version 2.1, January 2011.

[22] C. Becker, H. Kulovits, M. Guttenbrunner, S. Strodl, A. Rauber, and H. Hofman, "Systematic planning for digital preservation: Evaluating potential strategies and building preservation plans," Int. Journal on Digital Libraries (IJDL), December 2009.

[23] DLM Forum Foundation, MoReq2010 - Model Requirements for Records Systems. Draft - v0.92, 2010.

[24] "IT governance institute. CobiT 4.1. framework - control objectives management guidelinces - maturity models," 2007.

[25] "IEEE 1540-2001 - IEEE Standard for Software Life Cycle Processes Risk Management," 2001.

[26] "ISO/IEC 27001:2005. Information technology - Security techniques Information security management systems - Requirements," 2005.

[27] J. Zachman, "A framework for information systems architecture," IBM Systems Journal, vol. 12, no. 6, pp. 276-292, 1987.

[28] C. Becker, G. Antunes, J. Barateiro, R. Vieira, and J. Borbinha, "Modeling digital preservation capabilities in enterprise architecture," in In 12th Annual International Conference on Digital Government Research (dg.o 2011), June 12-15, College Park, MD, USA., 2011. 